\title{
EL BUSCADOR DOCUMENTAL ARIAE: FUENTE DE INFORMACIÓN SOBRE REGULACIÓN DE LA ENERGÍA EN IBEROAMÉRICA
}

\author{
José-Antonio Sánchez-Montero, Liliana M. Melgar-Estrada y Gabriella \\ De-Stefano-Convit
}

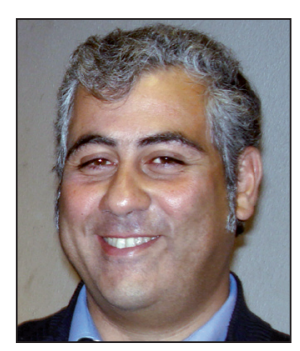

José-Antonio Sánchez-Montero es documentalista especializado en economía y energía, máster en información y documentación por la Universidad Carlos III de Madrid. Jefe de Área de Documentación de la Comisión Nacional de Energía (CNE).

Comisión Nacional de Energía Alcalá, 47. 28014 Madrid, España jsm@cne.es

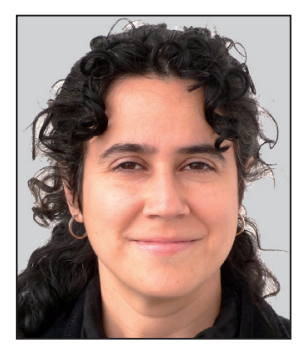

Liliana M. Melgar-Estrada es bibliotecóloga por la Escuela Interamericana de Bibliotecología de la Universidad de Antioquia (Medellín-Colombia), graduada del Máster Internacional en Bibliotecas Digitales (DILL). Actualmente es estudiante de doctorado de la Universidad Carlos III de Madrid.

Universidad Carlos III de Madrid Depto. de Biblioteconomía y Documentación Madrid, 126. 28903 Getafe (Madrid), España lilimelgar@gmail.com

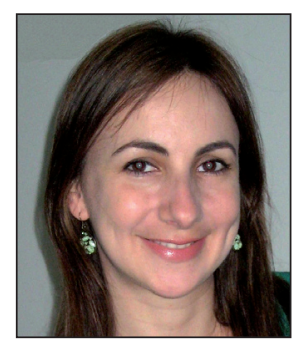

Gabriella De-Stefano-Convit es bibliotecóloga por la Universidad Central de Venezuela, estudiante del máster en Bibliotecas y Servicios de Información Digital de la Universidad Carlos III de Madrid, documentalista en la Universidad Simón Bolívar.

Universidad Simón Bolívar Valle de Sartenejas. Baruta, Edo. Miranda Apartado 89000, Caracas, Venezuela gabidestefano@gmail.com

\section{Resumen}

Ariae es la Asociación Iberoamericana de Entidades Reguladoras de la Energía, e integra los organismos de 19 países iberoamericanos. Se describe el servicio de información consistente en una biblioteca digital que recopila y pone a disposición de los miembros de la asociación y del público en general la documentación de acceso libre producida en el ámbito de sus actividades. Se describe Ariae y la motivación para la creación del servicio; se describen servicios de información similares en el ámbito energético y se detallan los objetivos y características del servicio de información de Ariae.

\section{Palabras clave}

Gestión de información, Servicios de información on-line, Derecho y regulación de la energía, Agencias reguladoras, Cooperación internacional, Transparencia administrativa, Asociaciones internacionales de reguladores de energía, Ariae.

\section{Title: Ariae search engine: information source on energy regulation in Iberoamerica}

\begin{abstract}
Ariae is the Iberoamerican Association of Energy Regulatory Entities, formed by the organizations of 19 Iberoamerican countries. In this article we present the new Ariae information service, consisting of a digital library that gathers and organizes the open access documents produced during the activities of the Association and its members. The article begins with an introduction to the Association and the motivations for creating the information service. Subsequently, it includes a brief presentation of similar services in the area, and finally a description of the objectives and characteristics of Ariae information service.
\end{abstract}




\section{Keywords}

Information management, On-line information services, Energy law and regulation, Regulatory agencies, International cooperation, Administrative transparency, International associations of energy regulators, Ariae.

Sánchez-Montero, José-Antonio; Melgar-Estrada, Liliana M.; De-Stefano-Convit, Gabriella. "El buscador documental Ariae: fuente de información sobre regulación de la energía en Iberoamérica". El profesional de la información, 2012, septiembre-octubre, v. 21, n. 5, pp. 537-541.

http://dx.doi.org/10.3145/epi.2012.sep.14

\section{Introducción}

La información es un valor fundamental para la toma de decisiones y es labor principal de los centros de documentación facilitar el acceso oportuno a las fuentes documentales.

En el sector de la regulación energética estos principios se ven acentuados por la necesidad de transparencia en la difusión de la información, lo que origina una producción alta y constante de documentos que las entidades responsables ponen a disposición de sus usuarios y pares. Por otro lado, la necesidad de cooperación entre dichas entidades plantea retos para el intercambio y difusión de su producción documental y/o informativa.

Se presenta el servicio de información documental de Ariae, la Asociación Iberoamericana de Entidades Reguladoras de Energía, que consiste en un sistema de acceso centralizado a los documentos producidos por las entidades energéticas reguladoras de Iberoamérica, creado con el fin de atender a las necesidades mencionadas, así como de favorecer la toma de decisiones informadas, la transparencia y la cooperación entre sus miembros.

Este servicio de información tuvo su origen en el Centro de Documentación de la Comisión Nacional de Energía de España (CNE). Fue presentado el día 22 de marzo de 2012 en el marco de la Asamblea General de la Asociación, celebrada en Cartagena de Indias (Colombia). Es un proyecto que, por su carácter público y abierto, el buscador Ariae se constituye desde un primer momento en una fuente de información esencial para el conocimiento del sector energético iberoamericano, y, en particular, de la regulación sectorial de energía. Es un servicio de interés tanto para el personal técnico de los organismos reguladores miembros de Ariae como para cualquier profesional o investigador interesado en la materia.

Está accesible en el sitio web de la CNE y desde la web de la Asociación.

http://www.cne.es/cgi-ariae/index.html

http://www.ariae.org/spanish/contenidos/serviciodocu mental.asp

\section{Motivación para la creación del servicio de información}

Desde 1997 Ariae integra los organismos reguladores de energía de 19 países iberoamericanos: Argentina, Bolivia, Brasil, Chile, Colombia, Costa Rica, Ecuador, El Salvador, España, Guatemala, Honduras, México, Nicaragua, Panamá, Perú, Portugal, República Dominicana, Uruguay y Venezuela, y al regulador del mercado centroamericano, la Comisión
Regional de Interconexión Eléctrica (CRIE) (Ruscalleda-Gallart, 2008).

http://www.ariae.org

Cada uno de estos organismos genera documentos y/o información producto de la actividad reguladora, que son publicados en sus respectivos sitios web de acuerdo con sus propios parámetros y tecnologías.

Al crearse la Asociación se observó la necesidad de compartir documentos e información entre los miembros. Para ello se consideraron varias alternativas. La más adecuada según las actuales posibilidades de los sistemas de información, habría sido la agregación automática de metadatos mediante el uso de estándares de interoperabilidad. Sin embargo, ante la disparidad de sistemas y la carencia de un estándar de metadatos y un vocabulario controlado común, se optó por la creación de una base de datos o catálogo colectivo administrado por documentalistas, integrando en una sola interfaz de búsqueda el acceso a las diferentes bases de datos o fuentes documentales existentes.

Por su carácter público y abierto, el buscador Ariae es una fuente de información esencial para el conocimiento del sector energético iberoamericano

\section{Servicios de información similares en el sector de la regulación energética}

Sin lugar a dudas, desde el punto de vista tecnológico, la Energy technology data exchange-World energy base (EtdeWeb) es la base de datos de información sobre energía más grande e importante a nivel internacional (Cutler, 2010). Según se indica en su presentación, en junio de 2012 EtdeWeb cuenta con 4.663 .000 referencias y 448.000 documentos a texto completo sobre bibliografía internacional especializada en energía. España es miembro fundador (Peset; Baiget, 2009), siendo el Ciemat la institución que coordina y supervisa su uso en ese país y el responsable de la selección, tratamiento documental y envío de la información energética producida en España a esta bases de datos, así como al sistema INS (INIS International nuclear information system/ International Atomic Energy Agency) (Ciemat, 2012). http://www.etde.org

Desde el punto de vista de la regulación energética, algunas asociaciones análogas a Ariae cuentan con servicios de información similares: 
The Council of European Energy Regulators (CEER-ACER) tiene en su sitio web un servicio que provee todos los informes anuales, nacionales, planes de trabajo, boletines de noticias y guías de buenas prácticas producidas por sus miembros. http://www.energy-regulators.eu/portal/page/portal/EER_HOME

La Energy Regulators Regional Association (ERRA) ofrece igualmente una biblioteca con una base de datos de los documentos producidos por sus integrantes, así como una base de datos de información tarifaria que permite hacer observaciones comparativas sobre tarifas de la energía en los países miembros.

http://www.erranet.org

La Energy technology data exchangeWorld energy base (EtdeWeb) es la base de datos de información sobre la energía más grande e importante a nivel internacional (Cutler, 2010)

La International Energy Regulation Network (IERN) cuenta con una sección de publicaciones en su web, en la que recoge una importante colección de recursos documentales a texto completo sobre regulación de la energía ordenados temáticamente.

http://www.iern.net/portal/page/portal/IERN_HOME/ IERN_ARCHIV/Publications

Además, cada uno de los 19 organismos reguladores de Ariae ofrece servicios de información documental en sus sitios web, incluyendo desde catálogos de enlaces hasta completos servicios de bibliotecas digitales.

Finalmente, hay dos servicios de destacada relevancia (Peset; Baiget, 2009): el DOE Information bridge y la Energy citations database.

\section{Objetivos del servicio de información de Ariae}

El principal objetivo de Ariae es promover el avance y el intercambio de experiencias regulatorias en los sectores de la energía, compartir el conocimiento regulatorio en dichos sectores y propiciar la comunicación entre especialistas y profesionales de las entidades asociadas que lo integran, así como facilitar la transferencia de conocimientos, informaciones, experiencias y estudios en los campos técnico, económico, normativo, jurídico y otros, aplicando modernas tecnologías de información (Canales-Nájera, 2008).

Mediante el diseño de un servicio de información online, el proyecto busca desarrollar estrategias para el cumplimiento de estos objetivos al facilitar a los miembros de la Asociación, en el marco de un repositorio único en internet, el acceso a los documentos producidos por Ariae y por los organismos reguladores miembros.

\section{Creación del fondo documental}

Al comenzar el proyecto ya existía una serie de recursos documentales dispersos en varias plataformas que fueron objeto de estudio para definir su inclusión en el proyecto:
- Página web de Ariae: documentación de reuniones anuales, cursos, seminarios y foros organizados, etc.

- Centro de documentación de la CNE.

- Sitios web de los organismos reguladores miembros de Ariae.

De acuerdo con la normativa sobre acceso y propiedad intelectual de la Asociación, definida en sus estatutos, durante 2011 se contactó con los miembros de Ariae para obtener la autorización de uso de los documentos seleccionados para aparecer en el servicio.

\subsection{Proceso de selección: fuentes y criterios}

El principal criterio de selección que se ha tenido en cuenta es que la información haya sido producida por Ariae o por las entidades reguladoras miembros. Dentro de este conjunto se establecieron prioridades temáticas y documentales:

\section{Temáticas:}

- práctica regulatoria;

- informes técnicos, económicos, normativos y jurídicos de los sectores energéticos;

- documentos sobre la historia del ente regulador, de los sistemas energéticos, legislación y/o sostenibilidad energética de cada uno de los países miembros;

- propuestas de las entidades reguladoras con relación a los procesos de revisión de tarifas y/o estructura de precios, supervisión de mercados y protección del consumidor, así como inspección de las actividades energéticas.

- documentos que dan cuenta de mejores prácticas y experiencias.

\section{Documentales:}

- libros en coedición y editados por las entidades reguladoras miembros de Ariae (indizados por capítulos);

- presentaciones de las reuniones anuales de Ariae, y documentación producida en ellas;

- anuarios, balances y resúmenes estadísticos de los sectores energéticos de cada país miembro;

- estudios;

- contenidos formativos (presentaciones en congresos, conferencias y seminarios, así como materiales de cursos y masters);

- acuerdos y tratados de cooperación energética;

- informes de actividad regulatoria;

- informes anuales de los entes reguladores;

- planes estratégicos;

- folletos divulgativos (cartillas, guías divulgativas);

Para la compilación de estas fuentes se han explorado, además de los sitios web oficiales de los reguladores y los fondos del centro de documentación de la CNE, los sitios web de ministerios y otros organismos oficiales de los países miembros de Ariae, así como revistas científicas.

\subsection{Tratamiento documental}

Una vez seleccionada la documentación de interés, se procedió a la catalogación y análisis documental detallado que permite el acceso a los documentos en la base de datos por temas y tipos documentales. La indización documental está basada en un vocabulario controlado propio, especializado en regulación de la energía, creado por el Centro de Docu- 
mentación de la CNE para la gestión de sus propias bases de datos documentales.

\subsection{Documentación incluida}

La base de datos contiene casi 1.500 documentos clasificados en 34 tipos documentales. Es importante resaltar que se puede acceder al texto completo de la mayoría de ellos, bien directamente (archivo cargado en la base de datos) o a través del url que se facilita en la ficha del registro.

\begin{tabular}{|l|c|l|c|}
\hline \multicolumn{1}{|c|}{ País } & n. docs. & \multicolumn{1}{c|}{ País } & n. docs. \\
\hline Argentina & 90 & Honduras & 29 \\
\hline Bolivia & 45 & México & 96 \\
\hline Brasil & 185 & Nicaragua & 22 \\
\hline Chile & 81 & Panamá & 38 \\
\hline Colombia & 105 & Perú & 136 \\
\hline Costa Rica & 37 & Portugal & 66 \\
\hline Ecuador & 45 & República Dominicana & 34 \\
\hline El Salvador & 27 & Uruguay & 55 \\
\hline España & 298 & Venezuela & 30 \\
\hline Guatemala & 74 & & \\
\hline
\end{tabular}

Tabla 1. Documentos en el buscador Ariae por países miembros $(01 / 06 / 2012)$

\section{El servicio de información}

Se basa en el sistema de gestión de bases de datos documentales del Centro de Documentación de la CNE. Bajo un motor de búsqueda que emplea el software BRS Search, se pretende conseguir una búsqueda eficaz y exhaustiva en todas las bases de datos que componen el buscador documental Ariae, proporcionando múltiples combinaciones y posibilidades de recuperación y filtrado de la información.

\section{Interfaz: modalidades de búsqueda y recuperación}

Búsqueda geográfica: a través de un mapa interactivo, se da acceso a la información existente por países (junto a CRIE y Ariae (figura 1).

Búsqueda sencilla: el sistema busca en los principales campos de la base de datos: título, autor, materias, país y resumen.

Búsqueda avanzada: permite combinar opciones de búsqueda de acuerdo con las tipos y temas. Los campos "Entidad reguladora", "Sector", "Materias" y "Descriptores" cuentan con tablas de ayuda con términos normalizados que facilitan la recuperación.

Búsqueda por tipo documental: distingue los tipos documentales detallados en el apartado 5.1 producidos por Ariae y por los distintos entes reguladores.

Filtrado de resultados: es posible filtrar los resultados obtenidos, lo cual es útil cuando se ha realizado una búsqueda
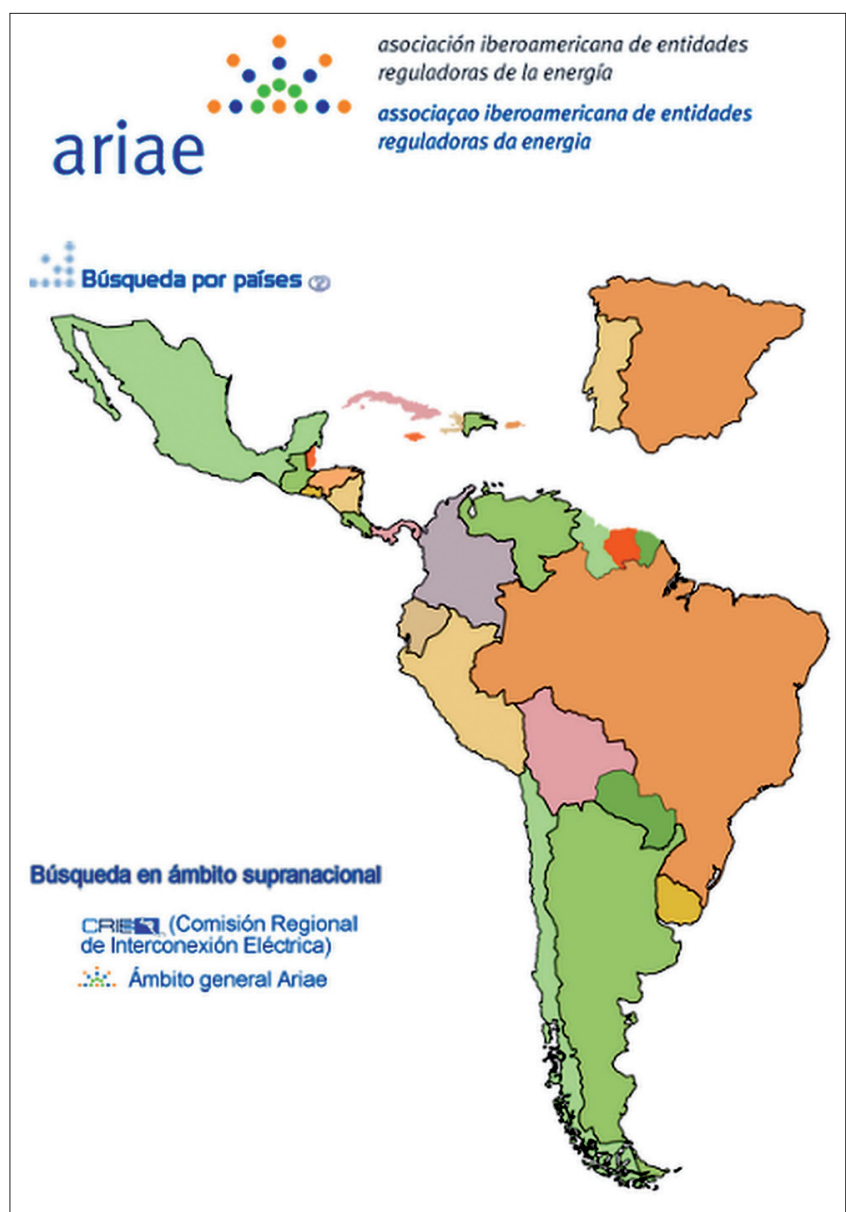

Figura 1. Búsqueda geográfica

poco precisa (por ej., sólo por país o tipo documental) (figura 3).

\section{Conclusiones y trabajo futuro}

El servicio de información documental de Ariae constituye un esfuerzo inicial en la vía del intercambio de información entre los miembros de dicha Asociación. Además, con el fin de continuar el trabajo de forma consensuada, se requerirá un trabajo para la creación de lenguajes documentales comunes (en español y portugués) así como de estándares de metadatos. La segunda fase del proyecto, que incluye la difusión entre los miembros de la Asociación, permitirá que el depósito de documentos en la base

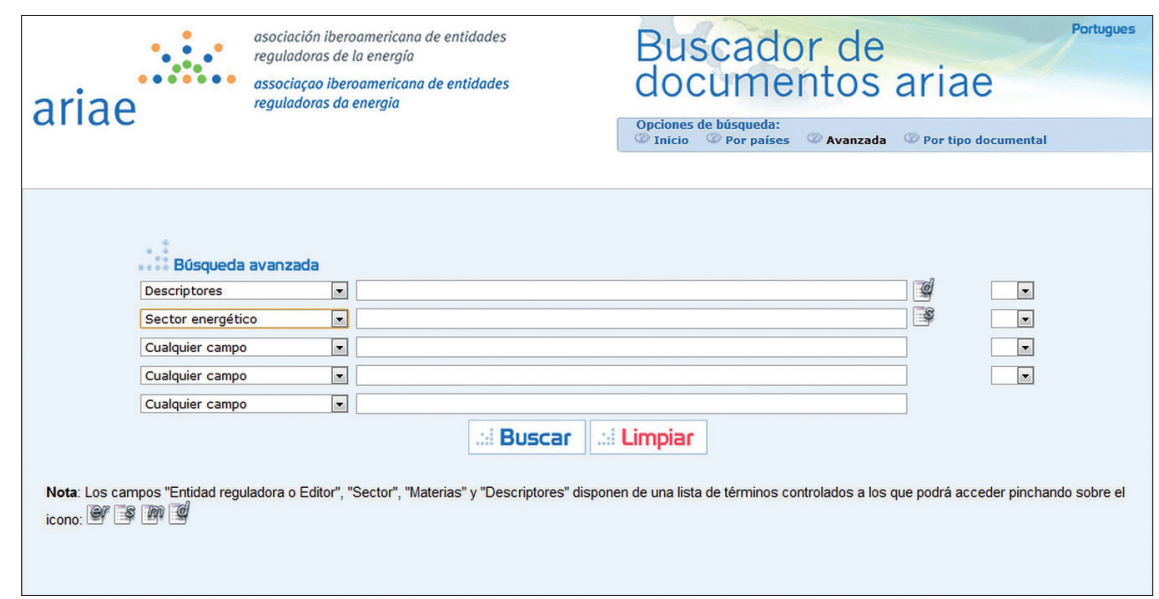

Figura 2. Búsqueda avanzada 


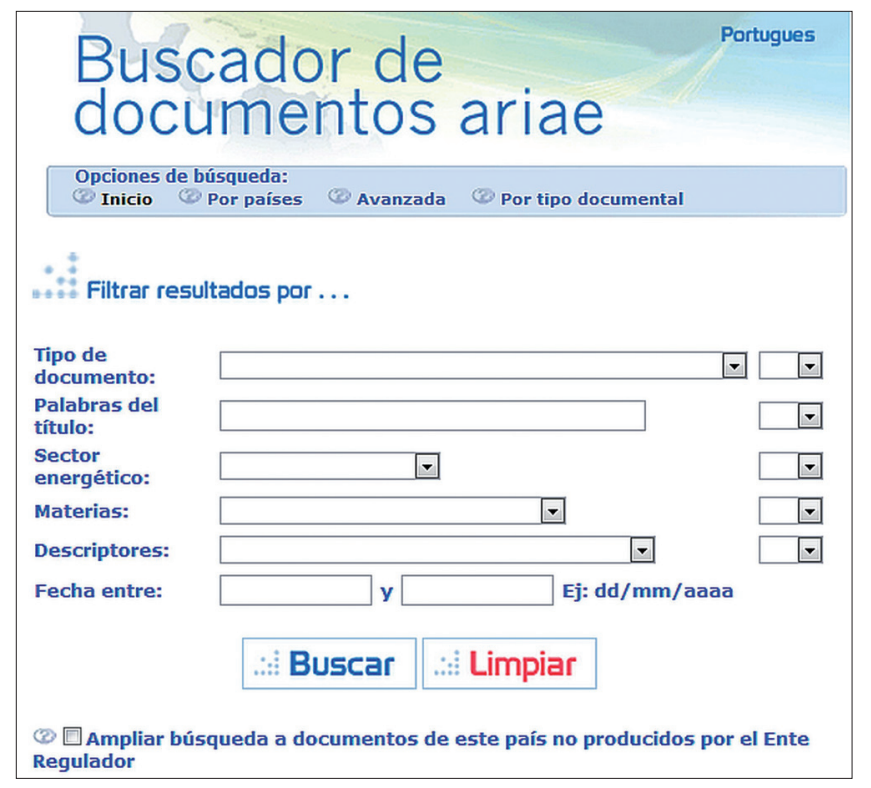

Figura 3. Filtro de resultados

de datos se haga en un futuro de manera descentralizada y automatizada.

Por otro lado, y siguiendo la propia idea inicial del servicio, Ariae se sumará a la tendencia actual en el tratamiento de la información en la Web de cumplimiento de estándares de publicación que permitan que los datos estén en abierto y enlazados.

\section{Bibliografía}

Canales-Nájera, Beatriz. "La Asociación Iberoamericana de Entidades Reguladoras de Energía (Ariae)". Revista de la Red de Expertos Iberoamericanos en Energía, 2008, 2o semestre, n. 3, pp. 24-27.

http://www.cne.es/cgi-bin/BRSCGl.exe?CMD=VEROBJ\&MLK $O B=425619361515$

Ciemat. Bases de datos de energía, 2012.

http://www.ciemat.es

Cutler, Debbie. "Database versus search engine". Research information, 2010, diciembre, v. 51, n. 29, pp.2-8.

http://www.researchinformation.info/features/feature. php?feature_id $=298$

Peset, Fernanda; Baiget, Tomàs. "OSTI-DOE, la fuente de información sobre energía. Entrevista a Karen Spence". EI profesional de la información, 2009, septiembre-octubre, v. 18 , n. 5, pp. 563-566.

http://dx.doi.org/10.3145/epi.2009.sep.12

Ruscalleda-Gallart, Sebastià. "El proceso de la regulación y la cooperación en Ariae. Retrospectiva de diez años y perspectivas de futuro". En: Energía y regulación en Iberoamérica. Thomson-Civitas, 2008, v. 2, pp. 371-376.

http://www.cne.es/cgi-bin/BRSCGI.exe?CMD=VEROBJ\&MLK $O B=370272444544$

\section{Máster en Buscadores: Marketing}

y posicionamiento.

Modalidad online. Ponte a punto

Recuperación de la información, marketing online innovador, programas de vigilancia tecnológica y de inteligencia competitiva

\section{Dirección: Cristòfol Rovira}

Calendario: diciembre 2012 - junio 2013

Más información: www.idec.upf.edu/mbusol

\section{PONTE A PUNTO}

Másters y Programas de postgrado

en Comunicación y Ciencias Sociales

Universitat Pompeu Fabra. www.idec.upf.edu

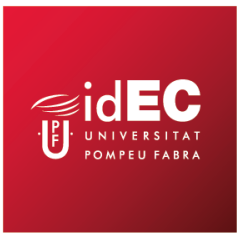

ÁREAS DE FORMACIÓN: Comunicación y Periodismo • Derecho • Gestión Cultural y Humanidades • Comunicación y Medios Audiovisuales • Política y Sociedad • Publicidad y Reputación Corporativa • Lenguaje y Traducción • Creatividad en Medios Digitales • Salud y Bienestar Social • Innovación y Gestión de la Información 


\section{MiBjb]jotecd}

\section{Actualidad bibliotecaria}

Fotografía momentos de lectura

\section{Opinión}

Recomendaciones de lectura Escolares

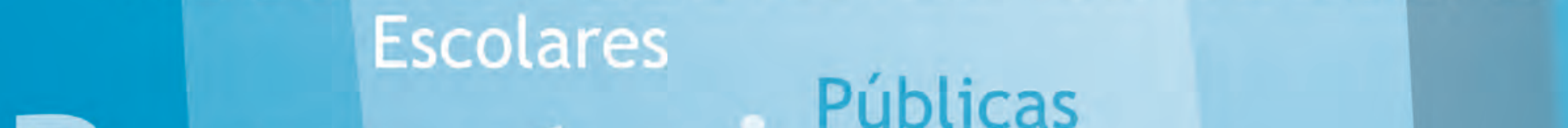

Reportaje Públicas Recursos Universitarias

Con pasado

Cartas a Mi B Bibliotecarios Con futuro

Noticias

Nuevas Tecnologías

Gestión documental

www.mibiblioteca.org 